\title{
RESEARCH OF THE PROBLEM OF OPTIMIZATION AND DEVELOPMENT OF A CALCULATION METHOD FOR TWO-STAGE CHAIN DRIVES USED IN HEAVY INDUSTRIAL VEHICLES IN CONDITIONS OF ECONOMIC EFFICIENCY
}

\author{
Sevda Aliyeva \\ Department of Mechanics ${ }^{1}$ \\ Javida Damirova \\ Department of Control and System Engineering ${ }^{1}$ \\ Sevinc Abasova $\bowtie$ \\ Department of Engineering and Computer Graphics ${ }^{1}$ \\ seva-abasova@mail.ru \\ ${ }^{1}$ Azerbaijan State Oil and Industry University \\ 27 Azadliq str., Baku, Azerbaijan, AZ1010
}

$\triangle$ Corresponding author

\begin{abstract}
The article is dedicated to the problem of optimization of chain drives of the drilling unit. At present, increasing the power per machine to the optimal limits, reducing the material and energy consumption per unit capacity of the machine, as well as operating costs are considered topical issues. The machines that are designed and constructed to optimal limits must be very powerful and productive. The machines that are applied to perform drilling works in the oil and gas industry must be easy to operate, reliable and have ability to operate for a long time. When constructing such machines, their being lightweight, economical, as well as their preparation in a short time and at low cost should be taken into account in advance. In order to ensure the reliable operation of drilling rigs, it is more expedient to apply chain drive in their mechanical transmission. First of all, the application of chain drive in drilling units and hoisting mechanisms is considered. Then a calculation method was developed for the chain drives of the drilling unit used in deep exploration wells and the exploitation of wells, and, accordingly, the calculation of the chain drive was carried out. The chain drive consists of drive and driven sprockets and a chain that encompasses the sprockets and engages in their teeth. Chain drives with several driven sprockets are also used. In addition to the basic listed elements, chain drives include tensioners, lubricating device and guards. The chain consists of hinged links that provide mobility or «flexibility» of the chain. Chain drives can be performed in a wide range of parameters. The calculation took into account the quality of the material, the service life and durability of the chain drive construction.
\end{abstract}

Keywords: gearbox, stars (sprockets), chain drive, objective function, two-stage, boring, durability, calculation method.

DOI: $10.21303 / 2461-4262.2021 .001930$

\section{Introduction}

The chain drive consists of the conducting sprockets (1) and sprockets being conducted (2), as well as the chain surrounding the sprockets (Fig. 1). Chain drive is an elastic coupling drive. The motion with these drives is transmitted to a certain distance by the adhesion force occurring among the sprockets [1-4]. Depending on their settings, chains are divided into transmission, traction and load chains. In general machine building, chain drive is mainly used for speeds up to $15 \mathrm{~m} / \mathrm{sec}$ and in load transmission up to $100 \mathrm{~kW}$. In special transmissions, the speed can be up to $35 \mathrm{~m} / \mathrm{sec}$, and the transmission force can be up to $1000 \ldots 3000 \mathrm{kw}$.

Thus, the following advantages of chain drive can be noted: it is possible to transmit motion over long distances through chain drive; the geometric dimensions of the drive are smaller than those of the belt drive; the average value of the drive number is constant; efficiency factor is high; it is possible to transmit energy to several driven shafts through one drive shaft; less force is applied to the shafts in the chain drive than in the belt drive [5]. 


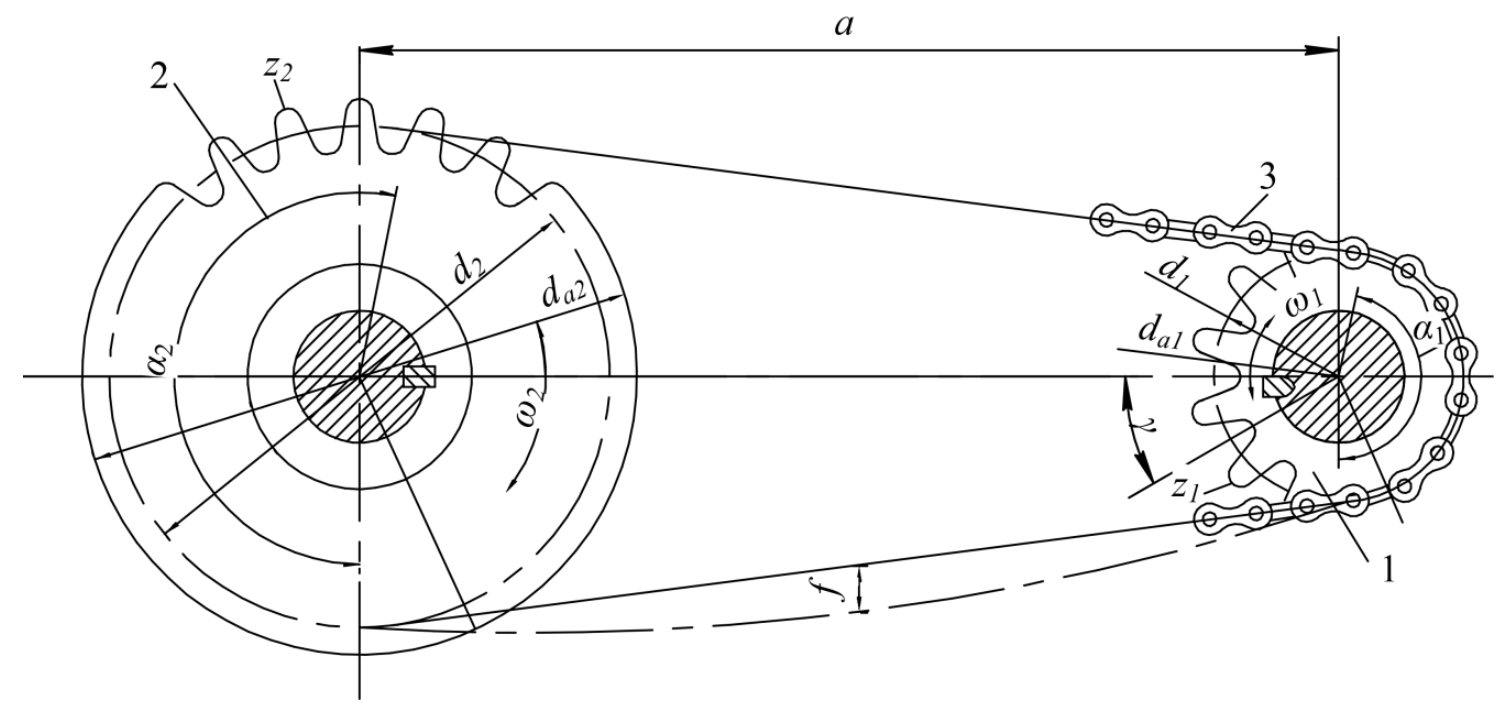

Fig. 1. Chain drive: 1 - conducting sprockets, 2 - sprockets being conducted, 3 - chain, $a$-inter-axis distance, $d_{1}$ and $d_{2}$ - diameter of the distribution circles of the conducting sprockets and sprockets being conducted, $d_{a 1}$ and $d_{a 2}$ - outer diameter of the conducting sprockets and sprockets being conducted, $z_{1}$ and $z_{2}$ - the number of teeth of the conducting sprockets and sprockets being conducted

Chain drives are widely used in agricultural and lifting-and-shifting machines, oil drilling equipment, motorcycles, bicycles, cars [6].

Chain drives are used: at medium center-to-center distances, at which gear drives require intermediate stages or parasitic gears, which are not caused by the need to obtain the desired gear ratio; when strict requirements for dimensions or when it is necessary to work without slipping.

In addition to chain drives, chain devices are used in mechanical engineering, that is, chain drives with working parts in conveyors, elevators, excavators and other machines.

In order to solve the optimization problem, the use of chain drive in drilling units and lifting mechanisms is considered.

\section{Materials and methods}

Machines and mechanisms produced in this day and age should have not only high efficiency, but also economic efficiency. The basis of these indicators should be established in the design process, the definition of construction parameters should be carried out by optimization and automatic design methods [7].

As the productivity speed and lifting capacity of machines and mechanisms increase, the resulting dynamic loads also increase, which should be investigated separately for each construction, depending on its purpose. In this regard, the optimal design and development of a new construction of the chain drive, which is widely used in the engineering industry to facilitate manual labor, is a topical issue.

Such problems as mechanics, dynamics, calculation methods, construction methods, testing of the chain drive have been considered by researchers. However, the problem of optimizing them in terms of economic efficiency has not yet been fully resolved.

The problems posed in the article were solved using mathematical methods of system dynamics and geometric programming method of optimization.

The statement of the problems on optimizing the construction of mechanical transmissions is to find such values of independent $x_{1}, x_{2}, \ldots, x_{m}$ variables in which the criterion for the optimization of the construction should obtain minimal values:

$$
g_{0}(\bar{x})=g_{0}\left(x_{1}, x_{2}, \ldots, x_{m}\right) \rightarrow \min ,
$$


at the same time the following restriction terms should be met:

$$
g_{i}(\bar{x})=g_{i}\left(x_{1}, x_{2}, \ldots, x_{m}\right) \leq 0
$$

where $a_{i} \leq x \leq b_{i} ; i=\overline{1, n} ; j=\overline{1, m}$.

In this case, such a solution is considered to be a compromise in which independent variables, on the one hand, obtain the values meeting all the restriction terms, on the other hand, the values meeting other terms, such as mass or cost, are accepted as the best values.

The feature of such problem statement is that the integer functions are contradictory, and therefore obtaining experimental values for all output parameters is impossible. For example, if the key indicator is the minimum value during the maximum service life, then the simultaneous meeting of these terms is contradictory to each other. In this case, a compromise solution must be accepted through the adoption of additional terms.

\section{Research results}

The optimally designed two-stage chain reducer has been developed in accordance with existing regulations and tested. Development of this reducer was carried out at «Baku Workers' Machine-Building Plant». The tests of a two-stage chain reducer used in production proved its having reliable efficiency. The economic efficiency of the new design was 650 USD per reducer.

The problem of accepting a compromise solution on the optimization of the construction is presented in the form of an optimization vector [8].

$$
\begin{gathered}
g_{i}\left(x_{1}, x_{2}, \ldots, x_{s}\right) \rightarrow \min , i \in I_{\min }, \\
g_{j}\left(x_{1}, x_{2}, \ldots, x_{s}\right) \rightarrow \max , i \in I_{\min }, \\
\phi_{i}\left(x_{1}, x_{2}, \ldots, x_{s}\right) \leq 0, i \in I_{\min }^{\prime}, \\
\phi_{j}\left(x_{1}, x_{2}, \ldots, x_{s}\right) \geq 0, j \in I_{2}^{\prime},\left|\frac{I_{\max }}{I_{\min }}\right|>2 .
\end{gathered}
$$

Chain drives are widely used in modern hoisting units appointed for drilling and repairing of oil wells.

The use of chain drives in hoisting units is explained by their specific advantages compared to such types of transmissions as belt and gear transmissions. One of these advantages is the possibility to transmit great powers at high rotational speeds and relatively large inter-center distances. An important feature in the exploitation of mechanical transmissions of hoisting units in the field (outdoor condition) is also the facilitation of assembling and disassembling of chain drives.

Therefore, the optimization of chain drives being widely used in the transportation of oilfield machines is a topical issue. In this regard, the problem on optimizing the chain drive of oil-field machines and mechanisms was stated [9].

The kinematic scheme of an ordinary two-stage chain reducer is considered to solve the optimization problem [10-12].

The functional dependence determining the value of a two-stage chain drive can be written as the sum of drives of chain values and star values. Then an objective function can be formulated as follows:

$$
g_{0}=C_{1}+C_{2},
$$

where $C_{1}$ is the value of sprockets mass of the chain drive; $C_{2}$ is the value of the line meter of the chain drive.

The sprockets mass on the chain drive mass can be determined by the following formula, taking into account the value of their preparation. 


$$
C_{1}=K_{1} m_{1}
$$

where $K_{1}$ is the value of chain transmission sprockets preparation, taking into account production costs; $m_{1}$ is the mass of sprockets.

The mass of the sprockets of a two-stage chain drive can be determined by the following formula in the first approximation:

$$
\begin{aligned}
& m_{1}=e \rho\left(V_{1}+V_{2}+V_{3}+V_{4}\right)=e \rho\left(\frac{\pi d_{1}^{2}}{4} B_{1}+\frac{\pi d_{2}^{2}}{4} B_{1}+\frac{\pi d_{3}^{2}}{4} B_{2}+\frac{\pi d_{4}^{2}}{4} B_{2}\right)= \\
& =\frac{\pi e \rho}{4}\left(d_{1}^{2} B_{1}+d_{2}^{2} B_{1}+d_{3}^{2} B_{2}+d_{4}^{2} B_{2}\right),
\end{aligned}
$$

where $\rho$ is the density of the whole material of the stars; $e$ is the filling factor of the sprockets volume, accepted in $e=0.7 \div 0.8$ limits; $d_{1}, d_{2}$ is the dividing diameter of the conducting and conducted $\left(d_{2}\right)$ sprockets of high-speed step; $d_{3}, d_{4}$ is the dividing diameter of the conducting $\left(d_{3}\right)$ and $\left(d_{4}\right)$ and conducted sprockets of the low-speed step; $B_{1}$ is the width of the chain drive of high-speed step; $B_{2}$ is the width of the chain drive of low-speed step.

Then, following expression can be written to determine the value of the sprockets:

$$
C_{1}=\frac{\pi K_{1} e \rho}{4}\left[B_{1}\left(d_{1}^{2}+d_{2}^{2}\right)+B_{2}\left(d_{3}^{2}+d_{4}^{2}\right)\right]
$$

The dividing diameter $(d)$ and width $(B)$ of the transmission chain are equal to the followings:

$$
d=\frac{p}{\sin \left(\frac{180^{\circ}}{z}\right)} ; B=i b
$$

where $p$ is step of the transmission chain; $b$ is the width of a single-row chain; $i$ is the number of rows; $z$ is the number of sprockets teeth. Then:

$$
C_{1}=\frac{\pi K_{1} e \rho}{4}\left\{i b_{1}\left[\left(\frac{p_{1}}{\sin \frac{180^{\circ}}{z_{1}}}\right)^{2}+\left(\frac{p_{1}}{\sin \frac{180^{\circ}}{z_{2}}}\right)^{2}\right]+i b_{2}\left[\left(\frac{p_{2}}{\sin \frac{180^{\circ}}{z_{3}}}\right)^{2}+\left(\frac{p_{2}}{\sin \frac{180^{\circ}}{z_{4}}}\right)^{2}\right]\right\} \text {. }
$$

The value of the transmission chain is equal to the followings:

$$
C_{2}=K_{2}\left(L_{1}+L_{2}\right)
$$

where $K_{2}$ is the value of the line meter of the transmission chain; $L_{1}$ is the length of the transmission chain of the high-speed step; $L_{2}$ is the length of the transmission chain of the low-speed.

$$
\begin{gathered}
L_{1}=W p_{1}=\left[\frac{z_{1}+z_{2}}{2}+\frac{2 a_{1}}{p_{1}}+\left(\frac{z_{2}-z_{1}}{2 \pi}\right)^{2} \frac{p_{1}}{a_{1}}\right] p_{1}=p_{1} \frac{z_{1}+z_{2}}{2}+2 a_{1}+\frac{p_{1}^{2}}{a_{1}}\left(\frac{z_{2}-z_{1}}{2 \pi}\right)^{2} \\
L_{2}=W p_{2}=\left[\frac{z_{3}+z_{4}}{2}+\frac{2 a_{2}}{p_{2}}+\left(\frac{z_{3}-z_{4}}{2 \pi}\right)^{2} \frac{p_{2}}{a_{2}}\right] p_{2}=p_{2} \frac{z_{3}+z_{4}}{2}+2 a_{2}+\frac{p_{2}^{2}}{a_{2}}\left(\frac{z_{3}-z_{4}}{2 \pi}\right)^{2},
\end{gathered}
$$

where $W$ is the number of links in the chain; $a$ is the inter-axis distance. 
Based on the above-mentioned explanation, the objective function can be written as follows to determine the minimum value of a two-stage chain drive taking $z_{1}=31-2 u$ and $z_{2}=u z_{1}$ into account:

$$
\begin{aligned}
& g_{0}=\left\{\frac { \pi K _ { 1 } e \rho } { 4 } \left\{i b_{1}\left[\left(\frac{p_{1}}{\sin \frac{180^{\circ}}{z_{1}}}\right)^{2}+\left(\frac{p_{1}}{\sin \frac{180^{\circ}}{z_{2}}}\right)^{2}\right]+i b_{2}\left[\left(\frac{p_{2}}{\sin \frac{180^{\circ}}{z_{3}}}\right)^{2}+\left(\frac{p_{2}}{\left.\left.\sin \frac{180^{\circ}}{z_{4}}\right)^{2}\right]}\right]+\right.\right.\right. \\
& \left.+p_{1} \frac{z_{1}+z_{2}}{2}+2 \cdot 40 p_{1}+\frac{p_{1}}{40}\left(\frac{z_{2}-z_{1}}{2 \pi}\right)^{2}+p_{2} \frac{z_{3}+z_{4}}{2}+2 \cdot 40 p_{2}+\frac{p_{2}}{40}\left(\frac{z_{4}-z_{3}}{2 \pi}\right)^{2}\right\} \rightarrow \text { min. }
\end{aligned}
$$

Strength reserve factor of chain drives is determined by the following formula according to $s$ calculation method:

$$
s=\frac{F_{\bar{p}}}{K_{3} F_{t}+F_{q}+F_{v}} \geq[s],
$$

where $F_{t}$ is a circular force, equal to:

$$
F_{t}=\frac{2 T}{d}=\frac{2 T \sin \left(\frac{180^{\circ}}{z}\right)}{p}=\frac{2 T \sin \left(\frac{180^{\circ}}{31-2 u}\right)}{p} .
$$

$T$ is a torque on the shaft of the conducting sprockets; $F_{q}$ is the load caused by chain oscillation; $F_{v}$ is the load caused by centrifugal forces; $F_{\bar{p}}$ is the destructive load for transmission chains; $[s]$ is the available value of the safety factor; $z$ is the number of the conducted sprockets teeth; $d_{1}$ is dividing diameter of the conducting sprockets; $p$ is transmission chain step; $k_{3}$ is the factor taking into account the dynamics of the load.

The calculation dependence for the determination of the destructive load $\left(F_{p}\right)_{\text {calculation }}$ was made depending on the value of the limit destructive loads, transmission roller chain step $p$ and the number of chain rows:

$$
\left(F_{p}\right)_{\text {calculation }}=0.083 \mathrm{ip}^{2.06} \text {. }
$$

Load $F_{q}$ resulting from the chain oscillation is determined by a known formula:

$$
F_{q}=k_{f} q i a g
$$

where $k_{f}$ is the oscillation factor, depends on the inclination angle of the chain drive and varies in limits $k_{f}=1 \div 6 ; q$ is the mass of the line meter of the transmission roller chain, depends on both step $p$ and the row of the transmission roller chain; $q$ can be determined according to the following formulas:

- for normal type of chains:

$$
q=i(0.385 t-8)
$$

- for heavy type of chains:

$$
q=i(0.438 t-9.3)
$$

$a$ is inter-axis distance of the chain drive, accepted within limits $a=(30 \div 50) \cdot p$ in the absence of restrictions on the inter-axis distance. 
The load caused by centrifugal forces $F_{v}$ can be determined by the following formulas:

- for normal type of chains:

$$
F_{v}=q_{1} v^{2}=i(0.385 p-7.98) \cdot\left[\frac{\pi n p}{30 \sin \left(\frac{180^{\circ}}{z}\right)}\right]^{2}=i(0.385 p-7.98) \cdot\left[\frac{\pi n p}{30 \sin \left(\frac{180^{\circ}}{z}\right)}\right]^{2}
$$

- for heavy type of chains:

$$
F_{v}=q_{1} v^{2}=i(0.438 p-9.3) \cdot\left[\frac{\pi n p}{30 \sin \left(\frac{180^{\circ}}{z}\right)}\right]^{2}=i(0.385 p-7.98) \cdot\left[\frac{\pi n p}{30 \sin \left(\frac{180^{\circ}}{z}\right)}\right]^{2} .
$$

Thus, taking into account the above-mentioned, the objective function for optimizing the parameters of two-stage chain reducers can also be presented as follows after the appropriate transformations:

$$
\begin{aligned}
& g_{o}=\left\{\frac{\pi K_{1} e \rho}{4}\left\{i b_{1}\left[\left(\frac{p_{1}}{\sin \frac{180^{\circ}}{z_{1}}}\right)^{2}+\left(\frac{p_{1}}{\sin \frac{180^{\circ}}{z_{2}}}\right)^{2}\right]+i b_{2}\left[\left(\frac{p_{2}}{\sin \frac{180^{\circ}}{z_{3}}}\right)^{2}+\left(\frac{p_{2}}{\sin \frac{180^{\circ}}{z_{4}}}\right)^{2}\right]\right\}+\right. \\
& \left.+p_{1} \frac{z_{1}+z_{2}}{2}+80 p_{1}+\frac{p_{1}}{40}\left(\frac{z_{2}-z_{1}}{2 \pi}\right)^{2}+p_{1} \frac{z_{1}+z_{2}}{2}+80 p_{2}+\frac{p_{2}}{40}\left(\frac{z_{4}-z_{3}}{2 \pi}\right)^{2}\right\} \rightarrow \min \\
& g_{1}=\frac{20 i_{1} e^{0.051 p_{1}}}{\left(\begin{array}{l}
2 K_{3} T_{1} \sin \left(\frac{180^{\circ}}{31-2 u_{1}}\right)+40 k_{f} q_{1} i_{1} p_{1}^{2}\left(0.385 p_{1}-7.98\right)+ \\
+i_{1}\left(0.385 p_{1}-7.98\right)\left[\frac{\pi n_{1} p_{1}}{30 \sin \left(\frac{180^{\circ}}{31-2 u_{1}}\right)}\right]^{2}
\end{array}\right)}>1, \\
& g_{2}=\frac{20 i_{2} e^{0.051 p_{2}}}{\left(\begin{array}{l}
2 K_{3} T_{3} \sin \left(\frac{180^{\circ}}{31-2 u_{2}}\right)+40 k_{f} q_{2} i_{2} p_{2}^{2}\left(0.385 p_{2}-7.98\right)+ \\
+i_{2}\left(0.385 p_{2}-7.98\right)\left[\frac{\pi n_{2} p_{2}}{30 \sin \left(\frac{180^{\circ}}{31-2 u_{2}}\right)}\right]^{2}
\end{array}\right)}>1, \\
& g_{3}=\frac{2.85}{p_{1}} \sqrt[3]{\frac{T_{1} k_{e}}{\left(31-2 u_{1}\right) k_{i}\left|\bar{p}_{1}\right|}}>1 \text {, } \\
& g_{4}=\frac{2.85}{p_{2}} \sqrt[3]{\frac{T_{3} k_{e}}{\left(31-2 u_{2}\right) k_{i}\left|\bar{p}_{2}\right|}}>1 \text {, } \\
& g_{5}=\frac{\left(31-z_{1}^{2}\right)\left(31-z_{3}^{2}\right)}{4 z_{1} z_{3}} \geq 1 \text {, }
\end{aligned}
$$

where function $g_{0}$ determines the minimum value of two-stage chain drive and becomes an objective function. Functions $g_{1}, g_{2}, g_{3}, g_{4}$ and $g_{5}$ characterize the strength reserve, wear resistance of joints, and kinematics of chain drive, and become limiting functions. 


\section{Discussion of experimental results}

Optimization of chain drives, widely used in oilfield drives of machines, is an urgent task. In this regard, the task was set to optimize the chain transmission of oilfield machines and mechanisms.

To solve the optimization problem, an idle two-stage kinematic diagram of a chain reducer is considered.

The problem of optimization of the design of one- and two-stage chain transmission has been solved. The minimum cost function is obtained two-stage chain transmission depending on the step, number of rows and section of gear values.

To determine the minimum value of a two-stage chain drive, taking into account $z_{1}=31-2 u$ and $z_{2}=u z_{1}$, the objective function is reflected in the analytical expression (1).

According to the calculation method, the safety factors of chain drives (2) and $F_{t}$ - circular force (3) are determined.

Depending on the value of the ultimate breaking loads, the pitch $\mathrm{p}$ of the transmission roller chain and the number of chain rows, the breaking load $\left(F_{p}\right)_{\text {calculation }}$ and the load $F_{q}$ from the chain oscillation are determined by expressions (4), (5), respectively.

According to analytical expressions (8), (9), the load from centrifugal forces $F_{v}$ was determined for normal and heavy chains, respectively.

As a result of the research, an objective function was formulated to optimize the parameters of two-stage chain gearboxes according to the analytical expression (10). The advantages of this study in comparison with those known on this topic are that it takes into account all the loads on the chain transmission. However, when the problem is solved using existing methods, the efficiency of the reducer decreases.

The chain drive has a large power transmission capacity. If the chain drives designed by the existing methods have large geometric dimensions, the chain drive designed by the proposed optimization method is considered having been improved. The function of the minimum value of two-stage chain drives is obtained depending on the chain step, the number of rows and the distribution of transmission numbers.

As a disadvantage of the study, the reduction in chain tension due to the impact of periodic forces as the drive operates can be noted.

In some cases, disruption of the normal operation of the chain due to the sudden impact of the loads in extreme conditions is inevitable.

\section{Conclusions}

Thanks to high capacity of power transmission of the designed chain reducer, reliable operation of oil mining machines operating with their application has been ensured.

Restrictions have been determined for the minimization function depending on the values of the transmitted loads, strength reserve, fatigue characteristic of the transmission chain and specific pressures in the joints.

The application of chain drives designed by the proposed method serves to increase productivity. The solution of the problem using the method of geometric programming 650 USD per reducer.

\section{Reference}

[1] Birger, I. A., Shor, B. F., Iosilevich, G. B. (1979). Raschety na prochnost' detaley mashin. Moscow: Mashinostroenie, 702.

[2] Semishin, I. M. (2003). Praktikum po metodam optimizatsii. Ul'yanovsk, 140.

[3] Huo, J., Yu, S., Yang, J., Li, T. (2013). Static and Dynamic Characteristics of the Chain Drive System of a Heavy Duty Apron Feeder. The Open Mechanical Engineering Journal, 7 (1), 121-128. doi: https://doi.org/10.2174/1874155x01307010121

[4] Childs, P. R. N. (2019). Belt and chain drives. Mechanical Design Engineering Handbook, 533-597. doi: https://doi.org/10.1016/ b978-0-08-102367-9.00012-3

[5] Suwannahong, W., Suvanjumrat, C. (2017). Analysis of Roller Chain Drive System with Multi-Flexible Body Dynamics Methodology. MATEC Web of Conferences, 95, 06007. doi: https://doi.org/10.1051/matecconf/20179506007

[6] Standard Handbook of Chains (2005). CRC Press, 432. doi: https://doi.org/10.1201/9781420027341 
[7] Djurayev, A., Mamakhonov, A. A., Yuldashev, K. K. (2019). Improvement of the Term of Service Life of the Drive Roller Chain of Transmission. International Journal of Advanced Research in Science, Engineering and Technology, 6 (3), $8508-8514$. Available at: http://www.ijarset.com/upload/2019/march/43-IJARSET-shohruz.pdf

[8] Alieva, S. Ya., Pashaeva, V. B. (2020). Determination of limitations for minimalizations depending on the main parameters of chain transmissions. Modern technologies in oil and gas engineering - 2020. Collected papers (proceedings) of the international scientific and technical conference, 312-314.

[9] Budynas, R. G., Nisbett, J. K., Shigley, J. E. (2011). Shigley’s Mechanical Engineering Design. McGraw-Hill.

[10] Motsoheyn, B. I. (1991). Elektro-tekhnicheskie kompleksy burovyh ustanovok. Moscow: Nedra, 254.

[11] Kerimov, Z. H. (2002). Machine parts, hoisting and transporting machines. Baku, 595. Available at: http://anl.az/el/k/kz_mhyqn.pdf

[12] Kerimova, I. M. (2019). On Possibility of Using a Toothed Belt Drive in a Drive to Rocker-Machines. 10th International Conference on Theory and Application of Soft Computing, Computing with Words and Perceptions - ICSCCW-2019, $763-767$. doi: https://doi.org/10.1007/978-3-030-35249-3_99

How to cite: Aliyeva, S., Damirova, J., Abasova, S. (2021). Research of the problem of optimization and development of a calculation method for two-stage chain drives used in heavy industrial vehicles in conditions of economic efficiency. EUREKA: Physics and Engineering, 4, 105-112. doi: https://doi.org/10.21303/2461-4262.2021.001930 